\title{
Comparative Study of Automotive Bumper with Different Materials for Passenger and Pedestrian Safety
}

\author{
Praveen Kumar.A ${ }^{1}$, Sameer Belagali ${ }^{2}$, Bhaskar ${ }^{3}$ \\ ${ }^{I}$ (M.Tech Automotive Engineering, VIT University, Vellore, India) \\ ${ }^{2}$ (M.Tech Automotive Engineering, VIT University, Vellore, India) \\ ${ }^{3}$ (Professor, SMBS, VIT University, Vellore, India)
}

\begin{abstract}
In this paper, the analysis is done on the automotive bumper to check the crashworthiness for the passenger safety. The bumper beam analyzed for the steel and composite material with the basic bumper design in the first phase, and then front part is modeled with the honeycomb and foam type in the second phase to compare the deformation and energy absorbed during the impact. A design of the automotive bumper must ensure the safety of passenger mean while, it needs to have low weight. With the role of safety, fuel efficiency and emission gas regulations are being more important in recent years that enforce the manufacturer to reduce the weight of passenger cars.

In this research, a front bumper beam made of steel and composite materials to provide more energy absorption in the structural form of honeycomb and foam type is assembled at the front of the bumper and is studied for impact modeling to determine the deformation and energy-absorption behavior.
\end{abstract}

Keywords: Bumper, Carbon fiber, Honeycomb, Impact analysis, Tensile strength

\section{Introduction}

Bumper beams are one of the important structures in passenger cars. For which we need to have careful design and manufacturing in order to ensure good impact behavior. The new bumper design must be flexible enough to reduce the passenger and occupant injury and stay intact in low-speed impact besides being stiff enough to dissipate the kinetic energy in high speed impact. The bumper beam is the key structure for absorbing the energy of collisions. Since, this is energy absorbing structure suitable impact strength is the main requirement for such a structure, this research analyses the parameters that directly affects impact characteristics and proposes easily achievable modifications resulting from impact modeling on commercial bumpers. A passenger car bumper is modeled and tested for the impact. With the introduction of automobile safety legislation, crashworthiness and safety should be considered as preconditions in light-weight design of bumper beam. There were four main strategic parameters being studied in the test modeling in the first step for metallic material. Firstly, the material i.e., how the type of material can affect the impact specifications and what kind of materials can be used as replacement in order to lower part weights. Steel and aluminum structures with a specified thickness that did not fail during the test depicted clearly that they are not suitable as bumper beam structure due to increasing weight. They increased the weight of the structure by nearly $500 \%$ and $100 \%$ respectively, in comparison with the composite bumper. To summarize, the objective of this research was to develop and propose a replaced composite bumper, which could satisfy following requirements:

a. Easy to manufacture by simplifying the shape. This was accomplished by removing strengthening ribs of bumper.

b. Being economical by utilizing low-cost composite materials.

c. Achieving reduced weight compared to the metallic bumpers.

d. Achieving improved or similar impact behavior compared to the current metallic structure.

Impact mechanics

It is important in the study of impacts to distinguish between the two different types of impacts that occur, elastic and plastic impacts. In an elastic impact a negligible amount of energy is lost between the two impacting bodies, for example, the collision of two billiard balls. A plastic impact involves a significant amount of energy dissipated in the collision. An impact between two vehicles or between one vehicle and a rigid body, where the vehicles crumple on impact, is an example of an elasto-plastic impact. The impacting phenomenon between an impactor and the front bumper in a low-speed full crash could be very complicated, since transient and nonlinear analyses are involved. But, in designing the front bumper, automobile manufacturers insist that the bumper system should not have any material crash or failure. Therefore, up to that point, the total energy is conserved throughout the impact duration. Since the impactor is assumed to be rigid and the bumper beam was made of metallic and composite material and shock absorber is a relatively low stiffness material, the distribution of the impact load is irregular along the contact area and over the contact region of the bumper. The bumper beam subjected to the impact load undergoes a constant deformation. 
An important consideration of momentum is that it can be neither created nor destroyed. Thus, the momentum before the impact is equal to the the momentum after the impact.

\section{Literature Review}

The analysis is done by considering the previous experiments carried out by the researchers in the field of selection of material in the automotive bumper impact analysis. Researchers focused on the mechanical properties of a hybrid glass epoxy composite for utilization in a passenger car bumper beam. A hybrid material, which is fabricated by modified SMC method present a good mechanical properties cause by applied pressure to yield a strong bond between the hybrid-reinforced fibers[1].

The design parameters for the bumper production such as cross section, longitudinal curvature, fixing method, rib thickness and the strength plays a significant role[2].

The researchers have studied on the study of impact behavior of vehicle to vehicle impact test and vehicle to barrier test in order to determine whether vehicle-to-barrier tests can serve as surrogates for vehicleto-vehicle tests in accident reconstruction [3].

The researches have been done on the study to develop a numerical analytical model of collinear low-speed bumper-to-bumper crashes, the model treats the car body as rigid structure and the bumper beam as a deformable structure attached to the vehicle. The model is also used to compare that how the structural characteristics of the vehicles' bumpers and the closing speed affect the crash pulse and to demonstrate a technique to estimate the maximum severity of a low-speed crash that has already occurred[4].

The experiments were done on the engineering thermoplastics which are used with good results in applications such as knee bolsters, structural instrument panels, head-impact-protection pillars, automotive bumpers, and body panels, where structural integrity, crashworthiness, and energy absorption capacity are key requirements [5].

\section{Analysis And Discussions}

Bumper of TATA 207 was considered and was modeled in PRO-e software. The modeling of three type automotive bumpers namely basic bumper model, second with foam material in front of the bumper and third one with the aluminum honey comb was modeled. The models were exported to ANSYS in .igs format for further analysis.

\subsection{The basic bumper with the steel}

The basic bumper design is modeled as shown in the fig. 2 with dimensions mentioned in the TABLE 1.

Table 1 Dimentions of bumper

\begin{tabular}{|l|l|}
\hline Radius of curvature(r) & $2380 \mathrm{~mm}$ \\
\hline Stay span length (L) & $1180 \mathrm{~mm}$ \\
\hline Young's Modulus (E) & $207000 \mathrm{~N} / \mathrm{mm}^{2}$ \\
\hline Sectional area (A) & $570 \mathrm{~mm}^{2}$ \\
\hline Moment of Inertia (Izz) & $248615 \mathrm{~mm}^{4}$ \\
\hline
\end{tabular}

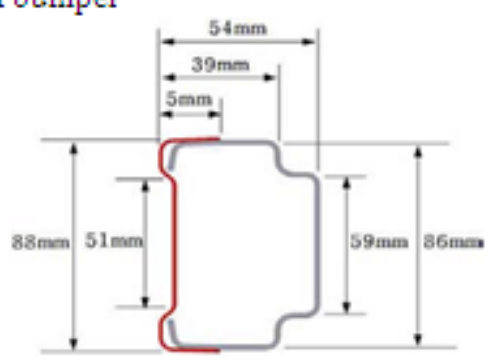

The imported bumper model is meshed which is having number of nodes 29878 and elements 14539. The meshed geometry is analyzed for the results of total deformation, stress and strain energy as explained below.

The properties of the steel are shown below in the TABLE 2.

Table 2 Mechanical properties of steel

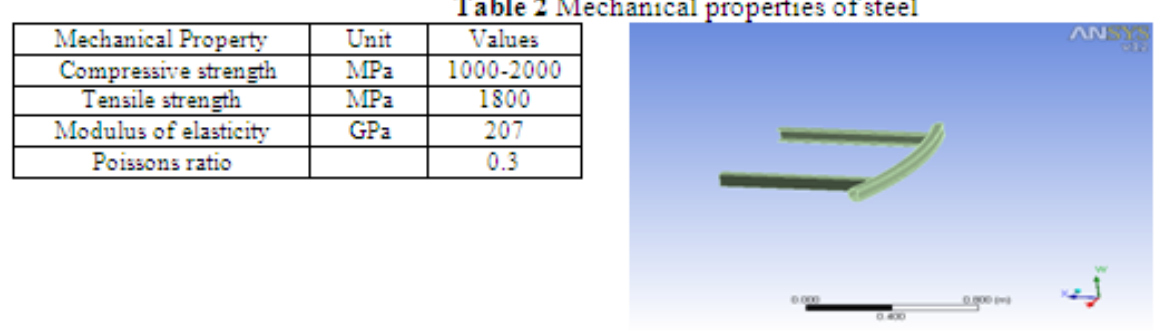

Fig.2 Basic bumper 
The mathematical calculation has to be done for the analysis i.e for the force input on the front bumper.

During impact energy absorbed by bumper is

$$
\mathrm{E}=0.5 \mathrm{mV}^{2}
$$

Where, $\mathrm{m}$ is the mass of the vehicle in $\mathrm{kg}$ i.e. $3000 \mathrm{Kg}, \mathrm{V}$ is the velocity of the vehicle in meter per second.

Since we are doing static structural analysis will consider the equation as

$$
\text { Work done }=\text { Force } \times \text { Distance }
$$

Here we have fixed the stopping distance as 1 foot and calculated the force, then we have

$$
\begin{aligned}
\text { Mass } \times \text { Acceleration }(\mathrm{g}) & =3000 \times 9.8 \times 8 \\
& =235200 \mathrm{~N}
\end{aligned}
$$

The value is applied on the front face and is solved for the results.

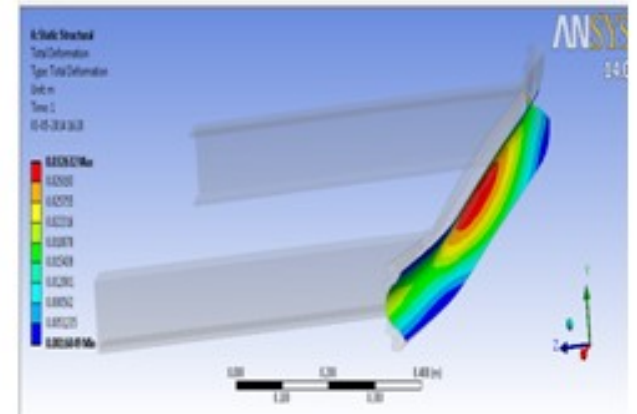

Fig.3 Deformation of basic bumper

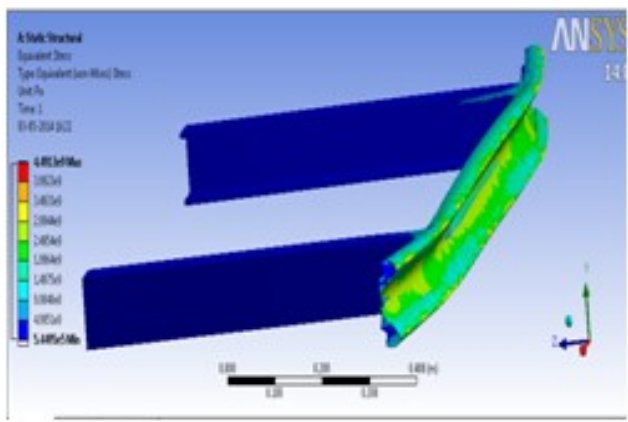

Fig.4 Stress analysis of basic bumper

As we know steel is known for its weight and it gives the rigid support to the vehicle, but as a bumper it should absorb the energy and it has to be stiff for the passenger safety. The theoretical aspect of steel it as the alloy of iron, with carbon which is contributing $2.1 \%$ of its weight carbon. Other elements and inclusions within iron act as hardening agents that prevent the movement of dislocations that naturally in the iron atom lattice. Varying amount of alloying elements retards the movement of their dislocations which in turn controls the tensile strength i.e. shown in the deformation fig. 3 and fig. 4 as it deforms to the value of $33 \mathrm{~mm}$ and the stress induced is more than the permissible tensile strength of the steel and is around $4.4 \times 10^{9} \mathrm{~Pa}$.

\subsection{Composite bumper}

The Dimensions are followed as same in the steel but the study of material is changed to carbon fibers. The mechanical properties of carbon fiber are shown in the table 3.

Table 3 Mechanical properties of carbon fiber

\begin{tabular}{|c|c|c|}
\hline Mechanical Property & Unit & Values \\
\hline Compressive strength & $\mathrm{MPa}$ & 110 \\
\hline Tensile strength & $\mathrm{MPa}$ & 110 \\
\hline Poissons ratio & & 0.77 \\
\hline
\end{tabular}

In the present condition of competitive world the fuel economy plays a important role so in order to achieve this the researchers are shifted towards light weight bumper such as composite material as carbon fiber is a material consisting of fibers about 5-10 micrometer in diameter and composed of carbon atoms. The carbon fiber is formed by arranging the carbon atoms bonded together in crystals that are aligned in parallel to the axis.

The geometry is meshed which gives the nodes 29882 and elements as 14538 . The analysis is done for the total deformation, stress and strain energy as explained below with the figures respectively. 


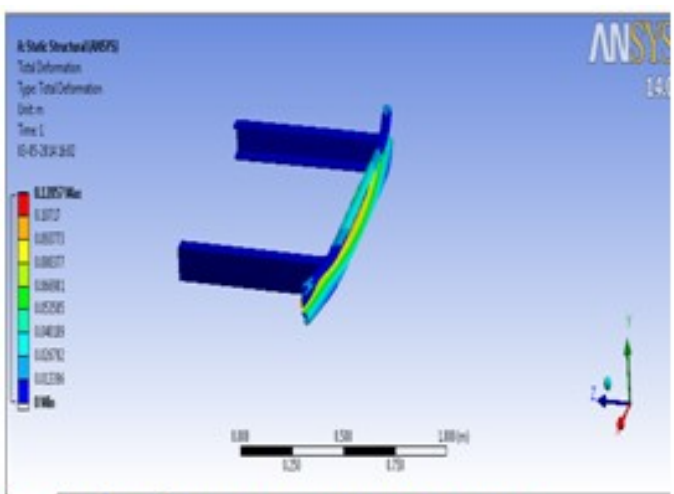

Fig. 5 deformation of carbon fiber bumper

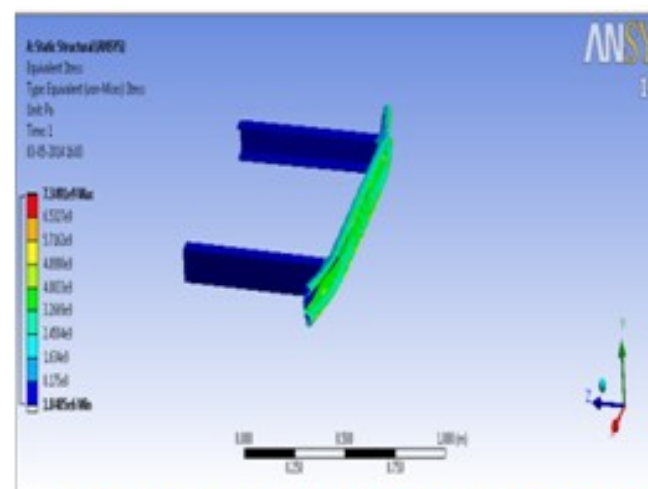

Fig.6 stress analysis of carbon fiber bumper

Coming to the bumper requirements since carbon fiber is having a rigid and brittle property so it crumples and reduces the harm to passenger. In the figure 5 deformation of carbon fiber is $127 \mathrm{~mm}$ and the maximum stress at the point is $7.391 \times 10^{9} \mathrm{~Pa}$ which exceeds the ultimate tensile strength of $110 \mathrm{MPa}$ and crumples as shown in fig.6.

The advantage of having carbon fiber as bumper is of high strength to weight ratio and because of brittleness it dissipates energy by breaking hence ensures passenger safety in comparison with the steel bumper.

\subsection{Bumper with the front part as foam}

The dimensions of the bumper are same but the front part of foam is modeled as shown in fig.7 on it to analyze the deformation and energy absorption. The front part of the bumper is designed to protect the pedestrian when accident takes place and it helps to absorb the energy.

The geometry is meshed which gives the number of elements 3613 as and nodes as 7660 . The meshed geometry is analyzed for the total deformation, strain energy and stress as explained below.

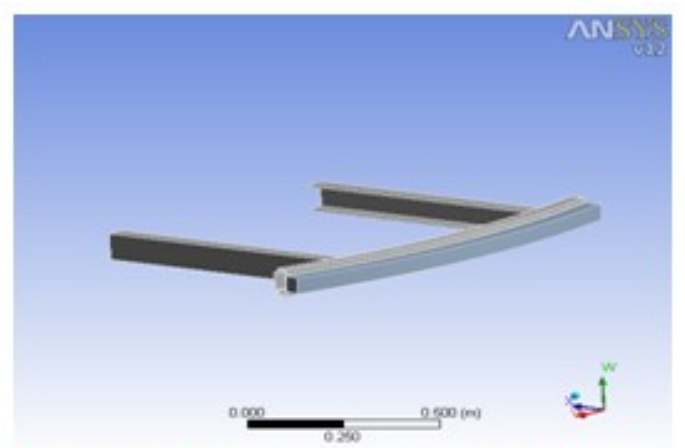

Fig. 7 Modified bumper with foam in front

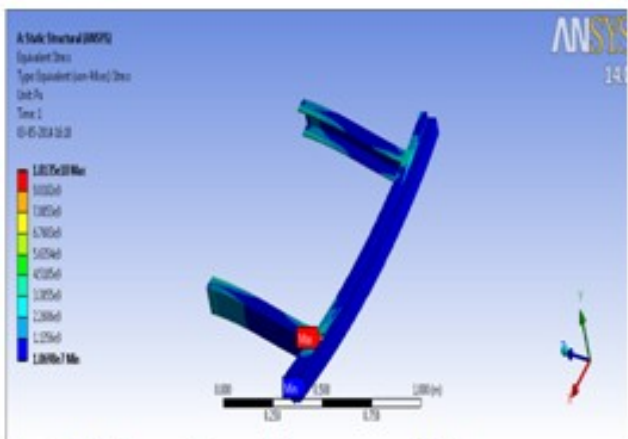

Fig.9 Deformation of bumper with foam

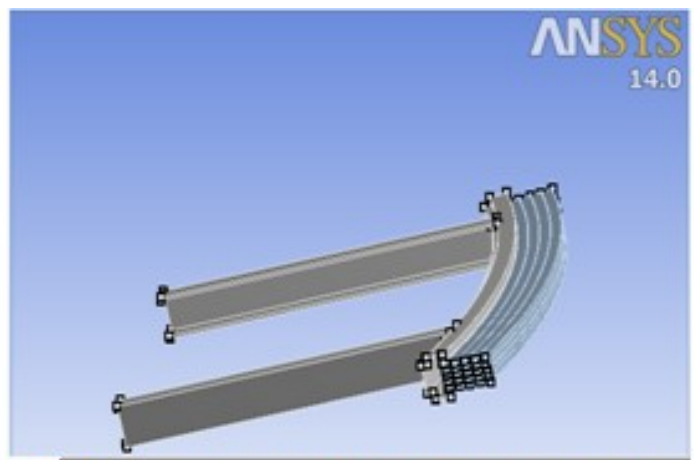

Fig.8 Modified bumper with honey comb structure

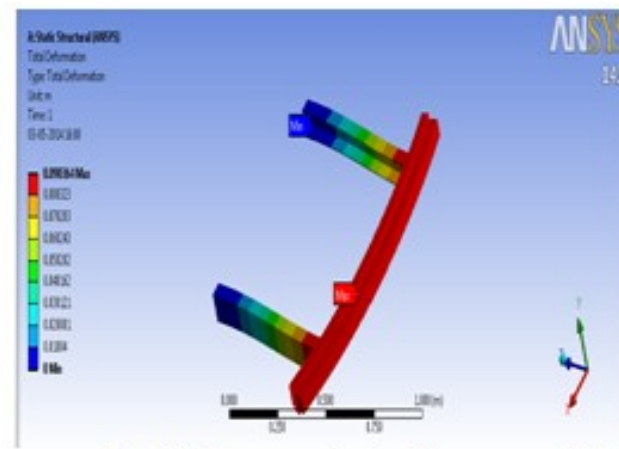

Fig.10 Stress analysis of bumper with foam

As we know closed-cell foams have not made of interconnected pores. The closed cell foams have good compressive strength and gives the deformation of $90 \mathrm{~mm}$ and reports maximum stress at $1.0135 \mathrm{X} 10^{10} \mathrm{~Pa}$ as shown in the fig.9 and fig 10 respectively. 


\subsection{Bumper with the front part as honeycomb}

The dimensions of the basic bumper remain the same but in the place of foam; honeycomb structure is designed to analyze the deformation and energy absorbed by the bumper i.e shown in the fig 8 .

The imported geometry is meshed with smooth grid elements which give the number of elements as 5405 and number of nodes as 16594. and is further analyzed for the total deformation, stress and strain energy absorption as shown and explained below.

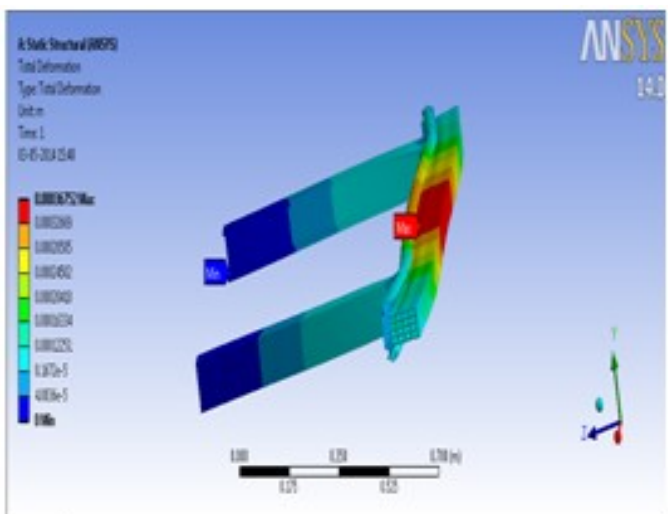

Fig.11 Deformation of bumper with honey comb

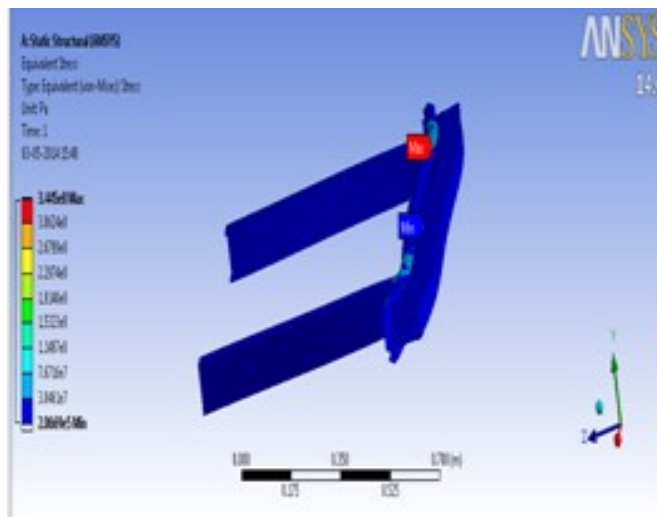

Fig.12 Stress analysis of bumper with honey comb

The honeycomb structure are characterized by considerable geometry by giving high crush stress by playing a role in crumple of car with the deformation of $0.367 \mathrm{~mm}$ and maximum stress of $3.445 \times 10^{9} \mathrm{~Pa}$ as shown in the fig 11 and fig 12 respectively.

\section{Conclusion}

The study of automotive bumper was done for three designs namely, basic bumper design with steel, with the attachment of foam and with the attachment of honeycomb and following conclusions were drawn.

1) The carbon fiber gives the high strength to weight ratio in comparison with the steel and dissipates energy.

2) The basic design with front part as a foam absorbs the energy and ensures the pedestrian safety,

3) The honeycomb structure having strength absorbs more energy hence reduces the chest ' $g$ ' thereby ensures passenger safety

By wisely inferring the above points we can to come to conclusion that by the utilization of carbon fiber, honeycomb and foam we can reduce shortcomings of normal bumper.

\section{Journal Papers:}

\section{References}

[1]. M.M. Davoodia,*, S.M. Sapuan a, D. Ahmad b , Aidy Ali a, A. Khalina b, Mehdi Jonoobi," Mechanical properties of hybrid kenaf/glass reinforced epoxy composite for passenger car bumper beam", material design 31 (2010) 4927-4932.

[2]. M.M. Davoodia,b, S.M. Sapuan b, A. Aidy c, N.A. Abu Osman a, A.A. Oshkour a, W.A.B. Wan Abas,” Development process of new bumper beam for passenger car", Materials and Design 40 (2012) 304-313.

[3]. Bradley E. Heinrichs, Jonathan M. Lawrence, Boyd D. Allin, James J. Bowler, Craig C. Wilkinson, Kurt W. Ising and David J. King MacInnis Engineering Associates," Low-Speed Impact Testing of Pickup Truck Bumpers". SAE Paper 2001-01-0893.

[4]. W. R. Scott, C. Bain, S. J. Manoogian, J. M. Cormier and J. R. Funk Biodynamic Research Corporation,” Simulation Model for Low-Speed Bumper-toBumper Crashes", SAE Paper 2010-01-051.

[5]. Daren Evans,TerryMorgans," Engineering Thermoplastic Energy absorbers for bumpers", SAE Paper 1999-01-1011 